\title{
Salud Mental, Función Docente y Mentalización en la Educación Preescolar
}

\author{
Ana María Mesa Ochoa \\ Pontificia Universidad Javeriana Cali - Colombia \\ Ana Cristina Gómez Arango \\ Pontificia Universidad Javeriana Cali - Colombia
}

\begin{abstract}
Resumo
Este artículo es de carácter teórico, su objetivo es presentar una revisión bibliográfica sobre la importancia de la promoción y prevención en salud mental desde el punto de vista de las intervenciones tempranas, que favorecen el desarrollo socio afectivo en contextos educativos. Subraya el vacío que hay al abordar los aspectos psicoafectivos de los maestros. Se parte de los objetivos de los programas de educación preescolar y el lugar de la competencia emocional del docente en ellos. Posteriormente, se presenta el concepto de mentalización, su relación con el desarrollo socio afectivo y la salud mental y, se enuncian programas que fomentan dicha capacidad en contextos tanto escolares como no escolares. Finalmente, se plantea una discusión alrededor de los programas de formación del docente de preescolar, su competencia emocional o capacidad de mentalización y la incidencia en el desarrollo socio emocional y la salud mental de los niños a su cargo.
\end{abstract}

Palabras-clave: Salud mental; educación preescolar; trabajo docente.

\section{Mental health, Teacher Function and Mentalization in Pre School Education}

\begin{abstract}
This is a theoretical paper, its aim is to present a literature review on the importance of health promotion and prevention in mental health from the point of view of early interventions, which promote children socio emotional development in educational contexts. We stress that there is a vacuum in addressing teachers psychological aspects. First, we present the aims of educative pre-school programs and the place teachers emotional competence have in them. Subsequently, we introduce mentalization concept and its relation with socio emotional development and mental health. We also illustrate some programs which have been designed to promote mentalization in school as well as non-school contexts. Finally, we present some ideas about preschool teachers' training programs and interventions that promote mentalization capacity. We demonstrate the relation between the promotion of socio-emotional development in the teachers and the positive incidence in mental health of the children with whom they work.
\end{abstract}

Keywords: Mental health; preschool education; teaching work. 


\section{Introducción}

Se ha demostrado que la intervención en promoción en salud mental durante la infancia, disminuye las alteraciones en el desarrollo e impide que se vuelvan patologías crónicas en Salud Mental (Lecannelier, 2006). La intervención temprana redunda en un incremento en la calidad de vida de quienes se ven beneficiados por dichos programas y en una reducción en los costos de los sistemas de salud, especialmente aquellos destinados al área terapéutica y de rehabilitación, pero también, en los del área de justicia y de seguridad social. Adicionalmente, incrementa las ganancias en los sectores económicos, por cuanto se dispone de una fuerza de trabajo más productiva (OMS, 2011).

Se asume que el desarrollo humano es un proceso mediado, asistido y guiado por los aspectos sociales en los que está inscrito. El avance en el conocimiento sobre la manera como procede el desarrollo humano y el reconocimiento de la importancia de la plasticidad cerebral característica de los primeros años, ha demostrado que los padres o cuidadores, así como los docentes, inciden de manera determinante en la maduración de las estructuras biológicas, fisiológicas y psicológicas. Es así como la educación preescolar ha pasado a ser una prioridad de los gobiernos, que buscan impulsar una formación de calidad para los niños en sus primeros años y promover la formación y actualización permanente de los docentes responsables de la educación preescolar (Mieles-Barrera, Henríquez-Linero, y Sánchez-Castellón, 2009). Es por ello que el objetivo de este trabajo es hacer una revisión teórica sobre los programas de capacitación a docentes de primera infancia, y específicamente, explorar el lugar que se le dan los aspectos psicoafectivos de los maestros.

\section{La salud mental y la etapa pre escolar}

La relación entre la salud mental y el preescolar está claramente establecida en la ley de primera infancia en Colombia. En síntesis, la ley presenta 5 razones por las que es imprescindible concederle un lugar prioritario a la atención a la primera infancia: 1) es la etapa más importante del desarrollo humano, 2) contribuye a mejorar el acceso y la permanecía en el sistema educativo, 3) incide positivamente en los procesos culturales y sociales, 4) genera una alta rentabilidad en términos económicos y, por todas estas razones, 5) contribuye a la disminución de las desigualdades sociales (Ministerio de Educación, 2011).

La educación preescolar, inicial o infantil, abarca los programas formales y no formales, a cargo de personal calificado, que se realizan en ambientes escolares o comunitarios. El Estado colombiano considera que la educación preescolar corresponde a los grados de pre jardín, jardín y transición, cuyo objetivo es brindar cuidado y educación fuera del ámbito familiar, a los niños y niñas entre tres y seis años (Mieles-Barrera, \& cols., 2009).
Los objetivos del nivel preescolar, establecidos en el artículo 16 de la Ley 115 de 1994, tienen en común que apuntan más a conquistas del desarrollo en términos de individualidad, autonomía y sociabilidad que a actividades o metas de carácter académico. Se puede decir que buscan favorecer el desarrollo integral del niño promoviendo la curiosidad por el conocimiento de sí mismo, del otro, del mundo en general y de las reglas que regulan su funcionamiento. Además, es el espacio propicio para preparar el ingreso del niño a los procesos de aprendizaje formal (Ministerio de Educación Nacional, 1994).

De acuerdo a lo anterior, Mieles-Barrera y cols. (2009) señalan que el preescolar es visto como un segundo escenario de socialización y de fortalecimiento afectivo, emocional y cognitivo de los niños y funciona como un sustituto del hogar porque las necesidades sociales y familiares propias del mundo contemporáneo así lo requieren. En consecuencia, a través de políticas gubernamentales, la sociedad ha implementado cambios en el funcionamiento de las instituciones educativas y en el papel de los docentes.

En lo que se refiere al currículo del preescolar, Jaramillo (s.f. b), señala que se deben tener en cuenta 5 dimensiones: 1) cognoscitiva, 2) socio afectiva, 3) comunicativa o del lenguaje, 4) estética, 5) espiritual y ética. La dimensión socio afectiva está presente en todas las actividades, sin embargo, hay momentos en que debe hacerse de manera propositiva.

Teniendo en cuenta que la escuela es un escenario privilegiado para favorecer el desarrollo emocional temprano, se han desarrollado programas que incluyen varios tipos de intervenciones por medio de las cuales se contribuye a que los niños adquieran las competencias necesarias para entender, manejar y expresar sus emociones e inscribirse en el mundo social (Gottfredson, \& Gottfredson, 2001 citados por Greenberg, Domitrovich, Graczyk, \& Zins, 2005; Elias, \& cols., 1997, citados por Greenberg, \& cols., 2005).

En cuanto a los programas dirigidos a los niños, Ross (1979) señala que algunos de los aspectos que se deben tener en cuenta con respecto al desarrollo emocional son el rol activo que tiene el niño en su propio desarrollo; la múltiple causalidad que requiere que se tengan en cuenta distintas teorías para su comprensión; el contexto que lo determina y al cual simultáneamente determina; el componente vinculante en torno a la cultura; el reconocimiento de la influencia que ejercen las tendencias del momento actual; la necesidad de variadas estrategias investigativas $\mathrm{y}$, por último, la interdependencia entre las investigaciones en desarrollo emocional y las políticas sociales.

En consonancia con lo anterior, el estudio de Bierman y cols. (2010), hace énfasis en que los programas de aprendizaje socio emocional pueden tener efectos preventivos importantes porque, si se implementan durante un tiempo considerable, influyen en la disminución de las tasas de agresión en la población, mejoran la competencia social y el compromiso académico de los alumnos en las escuelas. Por su parte, Denham, Zoller y Couchoud (1999), destacan que los procesos de socialización que incluyen explicaciones 
sobre las emociones y respuestas positivas y negativas del adulto hacia las experiencias emocionales del niño, inciden en el reconocimiento y comprensión que éste puede tener de las emociones.

El programa desarrollado por Denham y Burton (2003) para el desarrollo de la competencia emocional en niños, busca inicialmente que el niño pueda entender y nombrar las emociones; ayudar al niño a poner los sentimientos en palabras y a nombrar los afectos en sí mismo y en otros, y reconocer las acciones que pueden causar las emociones Este programa trabaja con la propia experiencia del docente en situaciones similares de reconocimiento de emociones, resolución de conflictos, etc. para que pueda tener una mejor comprensión de la vida emocional de los niños. Adicionalmente, presentan programas de otros autores que buscan incidir en la competencia emocional de los niños (Burton, \& Denham, 1998; Domitrovich, Cortes, \& Greenberg, 2002, Izard, \& Bear, 2001, Webster-Stratton, 2000, citados por Denham y Burton, 2003). En general, todos los programas presentados por los autores, coinciden en aspectos centrales como son 1) la importancia del apego de los niños con sus docentes como base para la intervención, 2) el trabajo sobre la comprensión y regulación emocional, 3) el abordaje de la resolución de problemas o conflictos sociales, y 4) un énfasis en la individualización y orientación positiva en el manejo de la conducta.

Ahora bien, si la relación entre el desarrollo emocional temprano y el contexto escolar parece estar clara, es importante entonces hacer una aproximación a la función del docente y a la relación que establece con sus alumnos. Desde esta perspectiva, tener en cuenta la competencia emocional del docente es de central importancia.

\section{La función del docente en pre escolar}

El docente de preescolar es fundamentalmente un promotor del desarrollo personal e integral de los niños, representa una figura sustituta de los padres o cuidadores y se convierte en referente de una autoridad protectora y afectiva (Mieles-Barrera, \& cols., 2009).

Con relación a la función del docente de preescolar Mieles y cols. (2009) señalan que cuando un docente sabe qué hacer y cómo, qué enseñar y a quiénes, cuáles son los métodos, las técnicas y las estrategias indicadas, esto sirve para favorecer el desarrollo de algunas competencias. Sin embargo, todo lo anterior resulta insuficiente para promover el desarrollo integral de los seres humanos y favorecer la salud mental. Para acceder a este logro es necesario que el docente pueda identificar sus propias necesidades y tener la capacidad de hacerse cargo de su experiencia emocional (Mieles-Barrera, \& cols., 2009).

El desarrollo de las habilidades emocionales del docente ha sido tema de interés investigativo. Algunas veces se le nombra con el término de inteligencia emocional (Cabello, Ruiz-Aranda, \& Fernández-Berrocal, 2010; Fernández, Palomero, \& Teruel, 2009; Sala, \& Abarca, 2002); otras, como la calidad de la relación con el alumno (O’Connor, \& McCartney, 2007); y otras más, se habla de la percepción emocional (Saft, \& Pianta, 2001). En términos generales todos los estudios se enfocan más en la relación interpersonal docente - alumno en la que se inscriben los procesos de aprendizaje, que en los aspectos propiamente académicos o de la técnica pedagógica. En esta línea, se encuentran los hallazgos de Saft y Pianta (2001), que muestran de manera puntual que cuando los niños y su docente son del mismo grupo étnico o raza, los conflictos reportados por el docente son pocos y de menor gravedad. Adicionalmente, observan que la actitud del niño en clase está directamente relacionada con la percepción emocional y la respuesta sensible del docente hacia él.

O'Connor y McCartney (2007) en su estudio sobre las interacciones entre el docente y el niño, llegan a tres importantes conclusiones: 1) existe una relación significativa entre la calidad de la relación y los logros alcanzados por el niño, 2) la relación positiva con el maestro protege al niño de los efectos negativos del apego inseguro con la figura materna y, 3) el efecto de la calidad de la relación maestro-alumno, está mediado por los comportamientos de ambos en el salón de clase.

En términos generales, según refieren Extremera y Fernández-Berrocal, 2004, Palomera, Fernández-Berrocal y Brackett, 2008, Poulou, 2005 (citados por Cabello, \& cols., 2010), los docentes reconocen la importancia que tiene la inteligencia emocional para el ejercicio de su profesión. En concordancia con ese planteamiento, se encuentran programas para trabajar en capacitaciones con ellos a ese nivel, como es el caso de El Laboratorio de las Emociones.

Se trata de un programa de la Universidad de Málaga (España) diseñado para favorecer la inteligencia emocional de los docentes. Consta de tres grupos de sesiones que en total duran 45 horas. En el primero, se da instrucción teórica presencial; en el segundo, los docentes deben poner en práctica lo aprendido con la tutoría virtual de sus profesores; y en el tercero, se vuelve a sesiones presenciales en las que se transmiten y comparten las experiencias vividas durante la práctica (Cabello, \& cols., 2010). En sentido general, los lineamientos de Fernández y cols. (2009) están de acuerdo con que la formación emocional del docente debe tener un componente teórico y otro vivencial. Los autores anotan que a pesar de la gran importancia que debería tener la competencia emocional del maestro, ésta sigue estando después de la del saber (el conocimiento) y de la del saber hacer creativamente (la técnica). Tanto Fernández y cols. (2009) como Abarca, Marzo y Sala, (2002), muestran cómo en la práctica se le da más importancia al aspecto académico de la formación de los niños que a la relación afectiva personal con ellos.

Con respecto a los programas que se ocupan de priorizar el componente psicoafectivo de la relación entre el docente y sus alumnos, Denham y Burton (2003) enfatizan que el punto de partida de cualquier intervención es crear un apego seguro, es decir, una relación emocionalmente positiva. Se debe trabajar el vínculo de la docente y sus figuras 
de apego e identificar el modelo interno de funcionamiento, para reconocer cómo se ve reflejado éste en la relación entre ella y los niños.

Se le invita a recordar, por ejemplo, con quien tenía buenas relaciones en la infancia y cómo eran, qué lo motivó a ser docente de pre-escolar, cómo fueron disciplinados en su infancia y las emociones dolorosas y negativas que vivieron con algunos métodos que utilizaron los adultos. Solo después será posible para el docente, pensar en algunas formas alternativas de hacerlo con los niños que tiene a su cargo. Los autores enfatizan cómo en los currículos tradicionales se asume que el desarrollo socio emocional se da implícitamente por el contacto de los niños con otros y se tiene la visión de que un buen profesor es quien mantiene su clase ordenada y disciplinada.

Adicionalmente, en la investigación de Reinke, Stormont, Herman, Puri y Goel (2011), se encontró que si bien los docentes perciben su responsabilidad en las intervenciones básicas en salud mental en el salón de clase, consideran que el aspecto socio emocional de sus estudiantes, es fundamentalmente responsabilidad de los psicólogos (Kratochwill y Shernoff, 2004, citados por Reinke, \& cols., 2011). La falta de formación en este sentido puede incrementar el estrés docente y dificultar el responder adecuadamente a los niños que presentan dificultades emocionales o de comportamiento, lo que se vuelve un círculo vicioso que incrementa el estrés del docente y las dificultades del niño (Yost, \& Mosca, 2002, citados por Sepulveda, Garza, \& Morrison, 2011).

En el contexto de la relación docente alumno, pensando en la importancia que tiene para el desarrollo del niño y particularmente para su salud mental, se encuentran investigadores que han mostrado que la capacidad de mentalización es fundamental porque contribuye a la comprensión de las relaciones interpersonales entre ellos. Adicionalmente, este concepto se presenta como componente esencial de algunas estrategias de intervención exitosas, tanto en el contexto escolar como no escolar, dirigidas a la promoción y prevención en salud mental (Allen, \& Fonagy, 2006).

\section{La mentalización y los programas que la promueven}

La mentalización es una capacidad que permite el reconocimiento de estados mentales en sí mismo y en los demás en términos de pensamientos, emociones, deseos e intenciones. Implica poder diferenciar el comportamiento visible y las posibles motivaciones o estados mentales que subyacen al mismo (Allen, \& Fonagy, 2006).

Este concepto se ubica en el campo de estudio del apego y el vínculo afectivo iniciado por Bowlby (1990) y continuado por Mary Ainsworth (1967) y Mary Main (2000), entre otros. Está en relación con términos pertenecientes al campo amplio de la psicología y el psicoanálisis como son los de empatía, posición depresiva y esquizoparanoide y mundo representacional.

La mentalización abarca el concepto de empatía (Twemlow, Fonagy, \& Sacco, 2005b), pero no se limita a éste porque, como lo explican Haslam-Hopwood, Allen, Stein y Bleiber (2006), la empatía corresponde al conocimiento y comprensión que se tiene de los sentimientos que pueden estar presentes en el otro. La mentalización en cambio, además de los sentimientos, incluye los pensamientos no solo en el otro, sino también en sí mismo.

La mentalización se relaciona con los conceptos de posición depresiva y esquizoparanoide de Melanie Klein (1935, 1948, citado por Stein, 2003); en la primera estaría presente, mientras en la segunda no.

Desde el punto de vista del mundo representacional la mentalización, según Lecours y Bouchard (1977, citados por Baretta, Roten, Lecours, Michel, \& Despland, 2006), es el proceso mediante el cual los afectos y los impulsos derivados de experiencias somáticas y motoras primarias, se trasforman en afectividad mentalizada, por medio de la adquisición y reorganización de las representaciones. Cuando la experiencia afectiva es de signo negativo, la elaboración mental se interrumpe y por lo tanto no puede ser asociada a otras, ni representada (González, Rodríguez, Sburlati, \& Triaca, 2000). La experiencia es escindida, rechazada y desplazada; se producen poderosas y dolorosas emociones. En este sentido, los autores señalan que la mentalización puede ser vista como la capacidad de modular, tolerar y elaborar mayores niveles de afectos negativos.

Para Haslam-Hopwood y cols. (2006), la mentalización no se enseña, pero se puede favorecer a través de programas de intervención, dirigidos a que los sujetos enfoquen su atención en ella para explicar fenómenos interactivos y experiencias subjetivas.

En el contexto escolar, la mentalización permite que el docente identifique y tenga en cuenta los múltiples sentidos que subyacen a las interacciones y afectan el aprendizaje (Twemlow, \& Fonagy, 2006). Le permite además, reconocer y regular su propia experiencia emocional (Allen, \& Fonagy, 2006).

El estudio de Twemlow, Fonagy y Sacco (2005a), plantea que el docente puede promover el desarrollo de la capacidad de mentalización del niño. De esta manera le ayuda a modular y regular los afectos negativos (González, \& cols., 2000), favorece el desarrollo integral y contribuye a la salud mental. En el mismo sentido O'Connor y McCartney (2007) señalan que la relación positiva con el docente protege al niño de los efectos negativos de un apego inseguro con sus padres o cuidadores.

Teniendo en cuenta la importancia de la mentalización, su relación con la salud mental y con la función del docente de preescolar, se presentan a continuación algunos factores que inciden en su desarrollo. Posteriormente se hace una revisión de los programas, individuales y grupales, que se han implementado en contextos no educativos y educativos para promover dicha capacidad.

En cuanto a los factores que inciden en el desarrollo de la capacidad de mentalizar en el niño, Fonagy, Target, Steele y Steele, (1998), señalan la calidad y el tipo de conversación que establece el adulto con los niños, los juegos 
de simulación en los que se involucran ambos y, por último, el manejo que el adulto le da a la disciplina.

Por otra parte, Fonagy (1999) considera que hay tres aspectos fundamentales en el desarrollo de la mentalización: 1) la especularización o reflejo diferenciado que hacen los padres de las emociones de los niños; 2) la parentalización reflexiva, a través de la cual el padre ayuda al niño a pensar sus emociones; y 3) el paso desde la equivalencia psíquica hacia la representación diferenciada entre mundo interno y externo.

Entre los programas encontrados para la promoción o recuperación de la capacidad reflexiva o mentalización en contextos no escolares, está el de Bleiberg (2006, citado por Allen, \& Fonagy, 2006), dirigido a profesionales de la salud mental en quienes se desencadenan crisis que afectan la mentalización, que es una capacidad esencial para el ejercicio de su labor terapéutica.

Otra propuesta de intervención es la de Haslam-Hopwood, Allen, Stein y Bleiber (2006, citado por Allen, \& Fonagy, 2006) dirigida a trabajar con pacientes psiquiátricos con patologías diversas. Se trata de un programa psicoeducativo que hace parte de las varias estrategias utilizadas para el acompañamiento en el proceso de reintegración a la vida fuera del hospital. Los autores advierten que no se ha realizado una investigación formal que dé cuenta de la efectividad específica de esta intervención. El objetivo es que los pacientes identifiquen y se pongan en contacto con estados mentales propios y de los demás. Además, trata de ayudarles a pensar de manera anticipada, posibles conflictos interactivos.

Algunas actividades utilizadas en el programa psicoeducativo de Haslam-Hopwood y cols. (2006, citados por Allen, \& Fonagy, 2006) son: 1) contar historias, a partir de imágenes del Test de Relaciones Objetales de Phillipson (Bellak,1996, citado por Haslam-Hopwood, \& cols., 2006, citados por Allen, \& Fonagy, 2006); 2) crear metáforas para comparar el significado y la emoción que intenta representar quien la produce, con la lectura de quienes la escuchan; 3) hacer descripciones de sí mismo centradas en los estados mentales; 4) inferir estados mentales a partir de la descripción de los hechos sucedidos en situaciones interactivas significativas; 5 ) inferir estados mentales a partir de la reflexión sobre eventos interactivos imaginados, que anticipan conflictos posibles en el futuro cercano; 6) representar interacciones conflictivas recientes con otros, desde diferentes perspectivas, a través de juegos de roles.

Otros programas en contextos no educativos son los citados por Slade $(2005,2006)$ para promover la mentalización de los padres hacia sus hijos. A través de ellos, se busca primero favorecer que el padre pueda ponerse en contacto y tolerar los estados mentales básicos del hijo y, posteriormente, que reconozca de qué manera inciden los estados mentales en la conducta y en otros estados mentales. Finalmente, que piense en los estados mentales propios y en los del hijo, que subyacen a las interacciones entre ellos (Slade, 2006). La autora describe dos programas. Uno dirigido a padres considerados normales, que se llevó a cabo en la modalidad de taller, a razón de una vez a la semana durante 3 meses; y el otro, con padres de alto riesgo psicosocial, durante dos años, a través de visitas domiciliarias.

En el contexto escolar propiamente dicho se encuentran dos programas muy interesantes por su relevancia social, diseñados por Twemlow y cols. (2005a); Twemlow Y cols. (2005b) para prevenir la violencia escolar. Ambos hacen parte del Proyecto de Pacificación Escolar. El primero se implementó en bachillerato y buscaba disminuir la posibilidad de que los estudiantes se expusieran a situaciones violentas potencialmente traumáticas. El segundo se realizó con estudiantes de primaria y pretendió evitar comportamientos violentos entre ellos. Los dos programas abordan el problema del matoneo teniendo en cuenta los participantes del conflicto, es decir, el agresor o matón, la víctima y el observador. Los autores consideran que el papel del profesor no es sólo de observador o de víctima, sino también de agresor. Señalan que uno de los objetivos fundamentales es involucrar al observador, que muchas veces es el profesor, en la identificación e interrupción de la violencia. Para ello, una de las estrategias centrales es incidir en la mentalización de los profesores. Los componentes del programa son: 1) Promover un clima positivo a través de una campaña publicitaria dirigida a crear conciencia de los estados mentales presentes en cada uno de los involucrados en la interacción violenta: agresor, víctima y observador; 2) implementar estrategias de manejo de la clase y planes de disciplina, para que frente a los problemas predomine una actitud mentalizadora por parte de los profesores, más que una orientación punitiva; 3) promover consejeros, que en la medida en que ofrecen una perspectiva diferente, contribuyen a la resolución de los conflictos; 4) realizar un programa de educación física llamado el guerrero gentil que busca enseñar a los estudiantes el uso de estrategias defensivas basadas en las artes marciales. De esta manera se pretende favorecer sentimientos de seguridad y protección básicos, que permiten acceder a alternativas cognitivas diversas, en aras de la solución de conflictos; y, por último, 5) utilizar un tiempo para la reflexión de más o menos diez minutos al final del día, durante el cual el grupo recuerda y piensa los episodios conflictivos de la jornada, teniendo en cuenta los tres roles involucrados en las interacciones violentas.

Otro programa para favorecer la mentalización es el de Apego, Mentalización, Auto-mentalización y Regulación afectiva (AMAR) propuesto por Lecannelier (2008) para el desarrollo del aprendizaje socio afectivo (ASA) en niños preescolares. El trabajo comprende cuatro etapas sucesivas. Se inicia con el apego como el vínculo básico que se debe fomentar entre el cuidador y el niño, continúa con la mentalización del cuidador, que debe tratar de entender los componentes mentales que subyacen al comportamiento del niño; y posteriormente, la auto-mentalización o la mentalización que le permite al docente de preescolar identificar lo que le sucede a él mismo a nivel de su mundo interno, especialmente frente a los comportamientos negativos o problemáticos del niño. Finalmente la regulación, que sería la intervención propiamente dicha por parte del adulto y que 
ayuda al niño a contener sus emociones, a calmarse y regular su conducta.

Para finalizar, se debe resaltar que Fonagy y Higgitt, 2004 (citados por Allen, Fonagy, \& Bateman, 2008), enumeran algunas de las características que tienen los programas efectivos para la promoción de la salud mental: 1) ser comprensibles, sistemáticos, estructurados y específicos con respecto a los factores de riesgo particulares; 2) darle más valor a la intensidad de la intervención que a la duración; 3) implementarse en las etapas más tempranas del desarrollo; e 4) incluir entrenamientos específicos para cada programa Denham y Burton (2003), enfatizan que promover influencias positivas es más efectivo que intentar contrarrestar las negativas.

\section{Discusión}

Como se observa en los planteamientos presentados, es clara la relación entre salud mental, desarrollo temprano y contexto pre-escolar, máxime en sociedades como las actuales en las que cada vez es más importante el lugar de los jardines infantiles en el desarrollo de los niños (Mieles-Barrera, \& cols., 2009).

Es importante resaltar que el contexto social y cultural es un factor central al abordar el tema del desarrollo emocional (Ross, 1979). En este sentido, el preescolar, como segundo escenario de socialización (Mieles-Barrera, \& cols., 2009) cumple diversas e importantes funciones que son asumidas por el Estado, tal como se puede ver en las políticas del Ministerio de Educación (1994), para regular el funcionamiento de las instituciones que trabajan con la primera infancia.

Una de las funciones principales del jardín infantil, tiene que ver con los procesos de socialización, tópico que se ha visto favorecido por el diseño y ejecución de intervenciones para el desarrollo socio afectivo.

Los programas para promover el desarrollo psicoafectivo, que se han realizado dentro o fuera de contextos escolares, han contribuido en la disminución de las tasas de violencia y han favorecido la elaboración de experiencias dolorosas (Bierman, \& cols., 2010; Twemlow, \& cols., 2005a; Twemlow, \& cols., 2005b ; Allen, \& cols., 2008), especialmente, las muy negativas, aquellas que no pueden ser asociadas a otras, ni representadas (González, \& cols., 2000), como es el caso de la exposición a una relación muy agresiva con la madre (Denham, \& cols., 1999).

El efecto positivo de estos programas amerita que se considere fundamental tratar de implementarlos en contextos deprimidos, donde son frecuentes las interacciones violentas a nivel social y familiar. De esta manera se puede promover la función reparadora y protectora del docente (O'Connor, \& McCartney, 2007) y tener efectos preventivos sobre la salud mental de la población infantil.

En el contexto preescolar tanto el docente, con sus conocimientos pedagógicos e historia personal, como el niño, con su propia historia y un psiquismo en desarrollo, entran en relación. A partir de la interacción cotidiana cons- truyen un vínculo. Cuando en esa relación predominan los sentimientos amorosos que dan cuenta del funcionamiento característico de la posición depresiva (Stein, 2003), se produce un impacto positivo en el desarrollo del niño, que redunda a nivel emocional, en conquistas en la individualidad, la autonomía, la sociabilidad y, en términos académicos, en la consecución de los logros pedagógicos del currículo. En este orden de ideas, se observa que varios autores han encontrado que una relación fundamentalmente positiva favorece la consecución de los logros del niño en la escuela (O'Connor, \& McCartney, 2007). Igualmente Saft y Pianta (2001) señalan que cuando existen similitudes culturales entre el docente y el niño, los conflictos son menos frecuentes y de menor gravedad.

Estos hallazgos se encuentran en el mismo sentido de los planteamientos de Burton y Denham, (1998); Domitrovich y cols., (2002); Izard y Bear, (2001), Webster-Stratton, (2000), citados por Denham y Burton (2003) y O'Connor y McCartney (2007), que consideran que el apego seguro entre el adulto y el niño, es un prerrequisito para llevar a cabo cualquier tipo de intervención que busque promover el desarrollo socio emocional en los niños.

Teniendo en cuenta lo anterior, atender el componente personal del docente es un factor del que no se puede prescindir, cuando se quiere promover el desarrollo emocional y la salud mental de los niños. Sin embargo, como lo señalan Denham y Burton (2003) en contradicción con esta idea, se observa que para la mayoría de los docentes el desarrollo de la competencia emocional de los niños se da de manera implícita, como resultado de sus interacciones cotidianas en el medio escolar. Por otra parte, se puede pensar que tampoco hay suficiente reconocimiento, por parte de los docentes, del protagonismo que tiene su experiencia emocional en el vínculo que se construye con los niños y la incidencia que eso tiene en su salud mental. Se sabe que puede ser positiva, cuanto se convierte en una relación reparadora, pero también negativa cuando predomina el funcionamiento esquizoparanoide, posición anti mentalización (Stein, 2003).

Si bien es cierto, los programas que buscan promover el desarrollo socioafectivo del niño de preescolar tienen estructuras claras, lineamientos específicos y actividades concretas para su aplicación (Fonagy, \& Higgitt, 2004, citados por Allen, \& cols., 2008; Denham, \& Burton, 2003), que buscan capacitar a los docentes para que cuenten con herramientas necesarias para saber el cómo y el qué hacer, se debe tener en cuenta que, como lo enfatiza Mieles-Barrera y cols. (2009), esto en sí mismo no garantiza la idoneidad de su aplicación. Para que estos programas puedan realizarse adecuadamente es esencial que los docentes se hagan cargo de sus propias experiencias emocionales especialmente las negativas y conflictivas, tal como lo anotan Allen y Fonagy (2006).

Los programas dirigidos a promover el desarrollo socio emocional de los niños que tienen en cuenta la experiencia emocional del docente, y los que se dirigen al desarrollo de la inteligencia emocional de los maestros, lo enfocan de dos maneras diferentes. Unos enfatizan el reconocimiento 
de la experiencia afectiva actual en la relación con el niño (Lecannelier, 2008) y en las experiencias emocionales presentes y cotidianas del maestro (Cabello, \& cols., 2010); otros en cambio, incluyen espacios donde se busca que a partir de la introspección, los docentes se pongan en contacto con experiencias infantiles relacionales con sus figuras de apego y puedan reconocer como podría estar incidiendo su historia en las relaciones que establecen con sus alumnos (Denham, \& Burton, 2003).

Teniendo en cuenta la importancia del docente, se considera que si bien es cierto los programas dirigidos a promover el desarrollo emocional de los niños son variados y presentan estrategias amplias para que los docentes los implementen, es necesario subrayar que sigue siendo esencial profundizar y enfocarse más en el desarrollo de estrategias que contribuyan al desarrollo de la capacidad automentalizante del docente (Lecannelier, 2008). Se sabe que si esta capacidad no se encuentra suficientemente elaborada, el docente puede continuar idealizando su función disciplinar, lo cual muchas veces impide el uso de mentalización como un recurso para entender y atender la experiencia emocional del niño y contribuir a su desarrollo socio emocional (Denham, \& Burton, 2003).

Ahora bien, ya que la promoción de la mentalización puede llevarse a cabo a través de estrategias individuales y grupales (Allen, \& Fonagy, 2006), se considera importante resaltar las intervenciones de tipo grupal pues permiten la reflexión colectiva, potencializan la interacción subjetiva, economizan posiblemente tiempo y esfuerzos y maximizan recursos. Se encontró que las estrategias son múltiples y creativas, comparten el hecho de que sirven para poner en acción reflexiones en torno a la propia mente, a la de los otros y, a la interacción entre ambas (Slade, 2005, 2006; Twemlow, \& cols., 2005a; Twemlow, \& cols., 2005b; Allen, \& cols., 2008).

En cuanto a los programas de mentalización propiamente dichos, se puede decir que la mayoría están dirigidos a trabajar en contextos no escolares; sin embargo, también se han implementado en el ámbito educativo. Los dos programas que hacen parte del Proyecto de Pacificación escolar, el de Twemlow y cols. (2005a) y el de Twemlow y cols. (2005b), se consideran muy interesantes y de gran importancia por su relevancia social, en la medida en que se ha demostrado que contribuye a la disminución de la violencia intraescolar. Adicionalmente, como un aporte importante está la inclusión de los docentes como participantes activos y no solo como observadores o ejecutores de la intervención.

Teniendo en cuenta que las intervenciones en salud mental tempranas son más efectivas porque tienen mayor impacto y son más rentables (Lecannelier, 2006, Fonagy y Higgitt, 2004, citados por Allen, \& cols., 2008), es clara la importancia que se le da al desarrollo y a la puesta en acción de programas que buscan favorecer la mentalizaciones en docentes y específicamente en el nivel pre escolar. Se considera que de esta manera se incide a nivel preventivo en salud mental, por las repercusiones positivas que de allí se derivan para las docentes como personas y para los niños con los que trabajan.
Algunos programas señalan la importancia de la mentalización pero priorizan los esfuerzos para entender la experiencia emocional del otro, bien sea en grupos de pacientes psiquiátricos, profesionales psicoterapeutas, estudiantes de primaria y bachillerato (Allen, \& Fonagy, 2006) como en padres de familia (Slade, 2005, 2006). Si bien este componente es esencial en la prevención y promoción de la salud mental, las autoras consideran que la reflexión y la elaboración de las experiencias emocionales del adulto que pretende favorecer el desarrollo emocional de los niños, llámese terapeuta, docente, etc., son una condición esencial. Adicionalmente, plantean que se deben incluir no solo en las vivencias actuales, como lo proponen muchos de los programas de desarrollo emocional y de mentalización, sino que se deben tener en cuenta las reflexiones sobre las relaciones primarias con las figuras de apego, tal como lo anotan Denham y Burton (2003). Las capacitaciones deben priorizar el desarrollo personal más que el entrenamiento en el uso de técnicas pedagógicas, porque a pesar de que los estudios coinciden en reconocer la importancia de la relación docente alumno (Bierman, \& cols., 2010; Denham, \& Burton, 2003; Saft, \& Pianta, 2001; O'connor, \& McCartney, 2007), en la práctica se le sigue dando más peso al alcance de los logros académicos (Fernández, \& cols., 2009; Abarca, \& cols., 2002) y a lo disciplinario (Denham, \& Burton, 2003).

Se ve claro entonces, que los cambios que se han dado en las instituciones educativas y en la función del docente de preescolar en los últimos años (Jaramillo, s.f.a), justifican que se dé prioridad a todos los esfuerzos dirigidos a la formación y capacitación de este último.

Se considera que en el perfil del docente de preescolar se le debe dar mayor importancia a la capacidad que tenga éste de mentalizar sobre sí mismo y sobre los demás. Se considera que si se encuentra suficientemente desarrollada, podrá hacer uso creativo de técnicas y estrategias de intervención que promueven el adecuado desarrollo de los niños $y$, por lo tanto, su salud mental, al mismo tiempo que los prepara para el ingreso al mundo del aprendizaje formal.

Por esta razón y para terminar, las autoras del presente artículo pretenden crear e implementar un programa para fomentar la automentalización de las experiencias infantiles, en grupo de maestras de primera infancia.

\section{Referencias}

Ainsworth, M. (1967). Infancy in Uganda: Patterns of Attachment Behavior Baltimore: Johns Hopkins University Press. Recuperado: 6 Nov. 2009. Disponível: http://www.psychology.sunysb.edu/ attachment

Abarca, M., Marzo, L., \& Sala, J. (2002). La educación emocional y la interacción profesor/a-alumno/a. Revista Electrónica Interuniversitaria de Formación del Profesorado, 5 (3). Recuperado el 15 abr. 2011. Disponível: http://www.aufop.org/ publica/ reifp/02v5n3.asp 
Allen, J., \& Fonagy, P. (2006). Handbook of Metallization-Based Treatment. London: John Wiley \& Sons, Ltd.

Allen, J., Fonagy, P., \& Bateman, A. (2008). Mentalizing in Clinical Practice. Washington, DC: American Psychiatric Publishing Inc.

Baretta, V., Roten,Y., Lecours, S., Michel, L., \& Despland, J. (2006). Mentalization of affects. An exploratory study of psychic elaboration and in-sesión expression of affects during a brief psychodinamic intervention for depression. Canadian Journal of Psychoanalysis 14 (2), 326- 349.

Bierman, K., Coie, J., Dodge, K., Greenberg, M., Lochman, J., McMahon, R., \& Pinderhughes, E. (2010). The effects of a multiyear universal social-emotional learning program. The role of student and school characteristics. Journal of Consulting and Clinical Psychology, 78 (2), 156-168.

Bleiberg, E. (2006). Treating professionals in crisis: A mentalizationbased specialized inpatient program. Em J. G. Allen, \& P. Fonagy (Orgs.). Handbook of Mentlaization-Based Treatment (pp. 233247). London: John Wiley \& Sons, Ltd.

Bowlby, J. (1990). El vínculo afectivo. Buenos Aires: Paidos.

Cabello, R., Ruiz-Aranda, D., \& Fernández-Berrocal, P. (2010). Docentes emocionalmente inteligentes. Revista Electrónica Interuniversitaria de Formación del Profesorado, 13 (1), 41-49. Recuperado: 15 abr. 2011. Disponível: http://www.aufop.com.

Denham, S., \& Burton, R. (2003). Social and emotional prevention and intervention programming for preschoolers. New York, NY: Kluwer Academic/Plenum Publishers.

Denham, S., Zoller, D., \& Couchoud, E. (1999). Socialization of Preschoolers' Emotion Understanding. Couchoud Developmental Psychology 30 (6), 928-936.

Fernández, M., Palomero, J., \& Teruel, M. (2009). El desarrollo socioafectivo en la formación inicial de los maestros. Revista Electrónica Interuniversitaria de Formación del Profesorado, 12 (1), 33-50. Recuperado: 15 abr. 2011. Disponível: http://www. aufop.com.

Fonagy, P. (1999). Persistencias transgeneracionales del apego: Una nueva teoría. Aperturas Psicoanalíticas, 3. Recuperado el 5, Mayo, 2011, de http://www.aperturas.org/3fonagy.html

Fonagy, P., Target, M., Steele, H., \& Steele, M. (1998). ReflectiveFunctioning Manual, version 5.0, for Application to Adult Attachment Interviews. London: University College London.

González, E., Rodríguez, M., Sburlati, M., \& Triaca, A. (2000, Octubre). Psicoanálisis, Prevención y Promoción de Salud. Una viñeta. Trabajo presentado en el XIV Congreso Latinoamericano de Psicoterapia Analítica de Grupo, Montevideo.
Greenberg, M., Domitrovich, C., Graczyk, P., \& Zins, J. (2005). The Study of Implementation in School-Based Preventive Interventions: Theory, Research, and Practice (Volume 3). DHHS Pub. No. (SMA). Rockville, MD: Center for Mental Health Services, Substance Abuse and Mental Health Services Administration. Recuperado: 15 abr. 2011. Disponível: http://prevention.psu.edu/ pubs/docs /CMHS_Implementation_report.pdf.

Haslam - Hopwood, G., Allen, J., Stein, A., \& Bleiber, E. (2006). Enhancing mentalizing through psycho-education. En J. G. Allen, \& P. Fonagy (Orgs.). Handbook of Mentalization - Based Treatment (pp. 249-267). England: John Wiley \& Sons, Ltd.

Jaramillo, L. (s.f.a). Antecedentes históricos de la educación preescolar en Colombia. Instituto de estudios superiores en educación. Unidad de nuevas tecnologías aplicadas a la educación. Universidad del Norte. Recuperado 15 Mai. 2011. Disponível: http://ylang-ylang.uninorte.edu.co:8080/drupal/files/ AntecendentesHsto ricosEducacionColombia.pdf

Jaramillo, L. (s.f.b). Aspecto legal para la organización de un centro escolar. Áreas o dimensiones curriculares. Instituto de estudios superiores en educación - Unidad de nuevas tecnologías aplicadas a la educación. Universidad del Norte. Recuperado: 15 Mai. 2011. Disponível:http://ylang-ylang.uninorte.edu.co:8080/drupal/files/ AreasDimensionesCurriculare s.pdf

Lecannelier, F. (2006). Estrategias de Intervención Temprana Preventivas en salud mental: Una experiencia Chilena en Investigación e Intervención. Ponencia: I Congreso Internacional de Actualizaciones en Psicología. Santiago de Cali. Cencolp.

Lecannelier, F. (2008). Apego y Aprendizaje Socio afectivo en la edad preescolar: evidencias y programas de intervención para el fomento de la alfabetización emocional. Revista Nacional de Psicopedagogía REPSI, 100 Santiago de Chile.

Ministerio de Educación Nacional (1994). Ley 115 de Febrero 81994. Ley General de Educación. Recuperado: 15 jun. 2011. Disponível: http://www.mineducacion. gov.co/1621/articles-85906_archivo_ pdf.pdf.

Ministerio de Educación Nacional (2011) ¿Por qué una política educativa para la primera infancia?. Recuperado: 21 jun. 2011. Disponível: http://www.mineducacion.gov.co/primerainfancia /1739/article-177832.html

Main, M. (2000). The organized categories of infant, child, and adult attachment: Flexible vs. inflexible attention under attachmentrelated stress. Journal of the American Psychoanalytic Association, 48 (4), 1055-1127.

Mieles-Barrera, M., Henriquez-Linero, I. \& Sanchez-Castellón, L. (2009). Identidad personal y profesional de los docentes de prescolar en el distrito de Santa Marta. Educación y Educadores, 12 (1), 43-59. 
O'Connor, E., \& McCartney, K. (2007). Examining Teacher-Child Relationships and Achievement as Part of an Ecological Model of Development. American Educational Research Journal, 44 (2), 340,369 .

Organización Mundial de la Salud (OMS, 2011). Salud y desarrollo del niño y del adolescente. Recuperado: 15 abr. 2011. Disponível: http://www.who.int/child_adolescent_health/topics/development/ es/index.html

Reinke, W., Stormont, M., Herman, K., Puri, R., \& Goel, N. (2011). Supporting Children's Mental Health in Schools: Teacher Perceptions of Needs, Roles, and Barriers. School Psychology Quarterly, 26 (1), 1-13.

Ross, P. (1979). Emerging themes for social-emotional development: Introduction to the section. American Psychologist, 34 (10), 930931.

Saft, E., \& Pianta, R. (2001). Teachers' perceptions of their relationships with students: Effects of child age, gender, and ethnicity of teachers and children. School Psychology Quarterly, 16 (2), 125-141.

Sala, J., \& Abarca, M. (2002). Las competencias emocionales de los futuros profesores/as. Revista Electrónica Interuniversitaria de Formación del Profesorado, 5 (3). Recuperado: 15 abr. 2011. Disponível: http://www.aufop.org/publica/reifp/02v5n3.asp
Sepulveda, C., Garza, Y., \& Morrison, M. (2011). Child Teacher Relationship Training.

Phenomenological Study. International Journal of Play Therapy. 20 (1), 12-25

Slade, A. (2005). Parental reflective functioning: An introduction. Attachment and human Development, 7 (3), 269-281.

Slade, A. (2006). Reflective parenting programs: Theory and development. Psychoanalytic Inquiry, 26 (4), 640-657.

Stein, H. (2003). Mentalization with reservations. Bulletin of Menninger Clinic, 62 (2), 143-149.

Twemlow, S., Fonagy, P., \& Sacco, F. (2005a). A developmental approach to mentalizing communities: I. A model for social change. Bulleting of the Menninger Clinic, 69 (4), 265- 281.

Twemlow, S., Fonagy, P., \& Sacco, F. (2005b). A developmental approach to mentalizing communities: II. The peaceful Schools experiment. Bulleting of the Menninger Clinic, 69 (4), 282-304.

Twemlow, S., \& Fonagy, P. (2007). Transforming violent social systems into non violent mentaliazing systems. An experiment in schools. Em J. Allen, \& P. Fonagy (2007). Handbook of Mentalization-Based Treatment. England: John Wiley \& Sons, Ltd.

\section{Sobre as autoras}

Ana María Mesa Ochoa (ammesa@javerianacali.edu.co)

Mg. Psicología Clínica, Pontificia Universidad Javeriana Cali

Ana Cristina Gómez Arango (acgomez@javerianacali.edu.co)

Mg. Psicóloga Clínica, Pontificia Universidad Javeriana Cali

Este artículo hace parte de la investigación Programa de intervención basado en la actividad referencial y la mentalización para maestros de niños de 4 a 6 años, del grupo Desarrollo Emocional y Salud Mental, financiado por la Universidad Javeriana Cali, Colombia. 